\title{
Using Convolutional Neural Network to Avoid Redundancy for Big Data at Data Centers
}

\author{
Mr. Abhishek Bari \\ Student ${ }^{1}$, \\ Department of Computer Engineering, \\ PCCoE, Nigdi, Pune, Maharashtra,India \\ Ms. Amruta Lahamge \\ Student ${ }^{2}$, \\ Department of Computer Engineering, \\ PCCoE, Nigdi, Pune, Maharashtra, India.
}

\author{
Ms. Dhanashree Jidge \\ Student ${ }^{3}$, \\ Department of Computer Engineering, \\ PCCoE, Nigdi, Pune, Maharashtra, India. \\ Ms. Komal Mane \\ Student ${ }^{4}$, \\ Department of Computer Engineering, \\ PCCoE, Nigdi, Pune, Maharashtra, India.
}

\author{
Prof. Pallavi Dhade \\ Assistant Professor, \\ Department of Computer Engineering, \\ PCCoE, Nigdi, Pune, Maharashtra, India.
}

\begin{abstract}
In the period of the Big Data a huge measure of gadgets and server farms stores or potentially produce different sort of information now and again for a wide scope of spaces and applications. In light of the idea of the area and application, these server farm will bring about large or quick/continuous capacity of information streams. Applying investigation over such information streams to find new procedure to make the space accessible. Lessening space issue will likewise assist with preparing the information a lot quicker. In this paper, we give an exhaustive review on utilizing a class of cutting edge AI procedures, in particular Conventional Neural Network to encourage the repetition evacuation in server farms. Excess records don't have a coordinating key and they contain blunders that may make repetition coordinating an exceptionally troublesome assignment. Copy records are presented as the aftereffect of absence of data or information, absence of standard arrangements, or there may be the any blend of these components. In this paper, we are going to introduce a through different examination of the writing on repetitive record location. We will cover similitude measurements which are generally in the used to recognize comparative field passages, and we present a broad arrangement of copy identification calculations that can distinguish roughly copy records in a database. We additionally spread different procedures for improving the productivity and versatility of rough copy discovery calculations. We finish up with inclusion of existing apparatuses and with a short conversation of the huge open issues in the region.
\end{abstract}

Keywords-- Duplicate records, Redundancy, Data focuses

\section{INTRODUCTION}

Information assume a significant job in current IT based economy. Numerous association and enterprises are absolutely subject to the precision of information to do there day by day to life. In this manner, there is need of value data put away in the server farms can have noteworthy ascent in cost suggestions to a framework that relies upon data to work and to direct the business. In a hazard free framework with entirely clear information, the development of an extensive perspective on the information comprises of connecting-in social terms, joining-at least two tables on their key fields. Shockingly, information regularly come up short on an interesting, worldwide identifier that would allow such an activity. Besides, the information are neither deliberately controlled for quality nor characterized in a predictable manner across various information sources. In this manner, information quality is frequently undermined by numerous components, including information section mistakes (e.g., Microsoft rather than Microsoft), missing trustworthiness imperatives (e.g., permitting passages, for example, Employee Age 1/4 567), and different shows for recording data (e.g., $44 \mathrm{~W}$. fourth St. versus 44 West Fourth Street). To compound the situation, in autonomously oversaw databases, the qualities, yet in addition the structure, semantics, and fundamental suppositions about the information may contrast too. Frequently, while coordinating information from various sources to execute an information stockroom, associations become mindful of expected methodical contrasts or clashes. Such issues fall under the umbrella-term information heterogeneity [1].

In spite of the fact that the general way to deal with repetition is shared by all stockpiling types, each stances explicit difficulties and prompts diverse exchange offs and arrangements. This assorted variety is frequently misconstrued, consequently thinking little of the significance of new innovative work. The principal commitment of this article is a characterization of repetition frameworks as indicated by six rules that relate to key structure choices: granularity, region, timing, ordering, method, and extension. This grouping distinguishes and depicts the various methodologies utilized for every one of them. As a subsequent commitment, we portray which blends of these structure choices have been proposed and discovered increasingly 
helpful for challenges in every capacity type. At last, remarkable examination challenges and unexplored plan focuses are distinguished and talked about.

\section{REPETITION DETECTION SYSTEM}

The programmed end of excess information in a capacity framework, normally known as repetitive, is progressively acknowledged as a powerful strategy to diminish capacity costs. In this way, it has been applied to various capacity types, including files and reinforcements, essential stockpiling, inside strong state drives, and even to arbitrary access memory

\section{CNN Steps:}

A Convolutional Neural Network (CNN) is called as multilayered neural system which have an exceptional design to discover complex highlights in any information or data. CNNs have been utilized in controlling vision in robots, record acknowledgment and for identifying copy information.

\subsection{Convolution}

A convolution is a consolidated incorporation of two capacities that gives you how one capacity changes the other.

$$
\begin{aligned}
(f * g)(t) & \stackrel{\text { def }}{=} \int_{-\infty}^{\infty} f(\tau) g(t-\tau) d \tau \\
& =\int_{-\infty}^{\infty} f(t-\tau) g(\tau) d \tau .
\end{aligned}
$$

There are significant things to make reference to in this procedure: the info record, the component finder, and the element map. The document being identified. The component identifier is a grid, normally $3 \times 3$ (it could likewise be $7 \times 7$ ). An element indicator is likewise alluded to as a piece or a channel.

\subsection{Apply the ReLu (Rectified Linear Unit)}

In this progression we apply the rectifier capacity to increment non-linearity in the CNN. Record are made of various items that are not direct to one another. Without applying this capacity the document order will be treated as a direct issue while it is really a non-straight one

\subsection{Pooling}

Spatial invariance is where the area of an article in a document doesn't influence the capacity of the neural system to distinguish its particular highlights. Pooling empowers the CNN to identify highlights in different document independent of the distinction in lighting in the photos and various points of the records.

There are various kinds of pooling, for instance, max pooling and min pooling. Max-pooling works by setting a grid of $2 \times 2$ on the component guide and picking the biggest incentive in that case. The $2 \times 2$ network is moved from left to directly through the whole component map picking the biggest incentive in each pass.

These qualities at that point structure another network called a pooled highlight map. Max pooling attempts to protect the primary highlights while likewise diminishing the size of the document. This lessens overfitting, which would happen if the CNN is given an excessive amount of data, particularly if that data isn't applicable in grouping the record.

\subsection{Flattening}

When the pooled highlighted map is acquired, the following stage is to straighten it. Leveling includes changing the whole pooled highlight map lattice into a solitary segment which is then taken care of to the neural system for preparing.

\subsection{Full Connection}

Subsequent to smoothing, the straightened include map is gone through a neural system. This progression is comprised of the info layer, the completely associated layer, and the yield layer. The completely associated layer is like the concealed layer in ANNs yet for this situation it's completely associated. The yield layer is the place we get the anticipated classes. The data is gone through the system and the mistake of forecast is determined. The mistake is then back propagated through the framework to improve the forecast.

\section{DATASET}

The dataset utilized in the experimentation here client input. Client input is put away in a registry on server. At whatever point client transfer the document it will be spared. At the point when we run the content to check the duplication we take all already inputted record.

\section{IMPLEMENTATION}

We separate records into organizers and give them their fitting names, i.e the preparation set and the test set. This makes it simpler to bring the records into Keras. Ensure that the working catalog has authorizations to get to the records

In this progression we have to import Keras and different bundles that we're going to use in building the CNN. Import the accompanying bundles:

- Sequential is utilized to introduce the neural system.

- Convolution2D is utilized to make the convolutional organize that manages the documents.

- MaxPooling2D layer is utilized to include the pooling layers.

- Flatten is the capacity that changes over the pooled highlight guide to a solitary section that is passed to the completely associated layer.

- Dense adds the completely associated layer to the neural system. 
Introducing the neural system

To introduce the neural system we make an object of the Sequential class.

\section{Convolution}

To include the convolution layer, we call the include work with the classifier item and go in Convolution2D with boundaries. The primary contention nb_filter. nbfilter is the quantity of highlight identifiers that we need to make. The second and third boundaries are measurements of the element identifier lattice.

\section{Pooling}

In this progression we decrease the size of the component map. For the most part we make a pool size of $2 \times 2$ for max pooling. This empowers us to lessen the size of the component map while not losing significant document data.

\section{Leveling}

In this progression, all the pooled highlight maps are taken and placed into a solitary vector. The Flatten work straightens all the component maps into a solitary segment.

\section{Full association}

The subsequent stage is to utilize the vector we acquired above as the contribution for the neural system by utilizing the Dense capacity in Keras.

\section{RESULTS AND DISCUSSIONS}

We present a class of productive models called Conventional Neural Network (CNN) for excess recognition. In this client needs to enlist first, when client is register he/she will get affirmation email. Utilizing the secret word in affirmation email client can continue further. Client will transfer the document from the site.

On the server side we have actualize the CNN algorithm once client transfer the record it is saved money on the server. When the record is moved it will be the contribution for the algorithm. With the assistance of following stream Convolutional Layer, Pooling Layer, Fully Connected Layer we will have the option to recognize if there is any excess .CNN learns the estimations of these records all alone during the preparation procedure. As opposed to decreasing the quantity of boundaries, for CNNs we force limitations on the model boundaries during preparing to shield them from learning the commotion in the preparation information. In spite of the fact that we despite everything need to determine boundaries, for example, filename and area. A CNN is in the least difficult case a rundown of Layers that change the volume into a yield volume.
We have checked our application for a little documents and huge records of 15 experiments with 6 copy record and 6 new ones. The precision we accomplished was $91 \%$. The things that should be viewed as further are the sound file, video documents.

\section{REFERENCES}

[1] Koolagudi, S.G. \& Rao, K.S. Int J Speech Technol (2012) 15: 99 https://doi.org/10.1007/s10772-011-9125-1 (Last referred on 5Nov-2019)

[2] Scherer, K. R. (2005). What are emotions? And how can they be measured? Social Science Information, 44(4), 695-729. https://doi.org/10.1177/0539018405058216 (Last referred on 5Nov-2019)

[3] http://practicalcryptography.com/miscellaneous/ma chinelearning/guide-mel-frequency-cepstral- coefficients-mfccs (Last referred on 5-Nov-2019)

[4] Brian McFee, Colin Raffel, Dawen Liang, Daniel P.W. Ellis, Matt McVicar, Eric Battenbergk, Oriol Nieto, "PROC. OF THE 14th PYTHON IN SCIENCE CONF. (SCIPY 2015)"

[5] S. Haq and P. J. B. Jackson, "Machine Audition: Principles, Algorithms and Systems," Hershey PA, 2010, pp. 398-423.

[6] Chollet, F., \& others. (2015). Keras. https://keras.io. (Last referred on 5-Nov-2019)

[7] Andy Liaw, \& Matthew Wiener (2002). Classification and Regression by randomForestR News, 2(3), 18-22.

[8] Chen, T., \& Guestrin, C. (2016). XGBoost: A Scalable Tree Boosting System. In Proceedings of the 22nd ACM SIGKDD International Conference on Knowledge Discovery and Data Mining (pp. 785-794). ACM. ISBN: 978-1-4503-4232-2

[9] Hearst, M. A., Dumais, S. T., Osuna, E., Platt, J., \& Scholkopf, B. (1998). Support vector machines. IEEE Intelligent Systems and Their Applications, 13(4), 18- 28. doi:10.1109/5254.708428 (Last referred on 5- Nov-2019) 